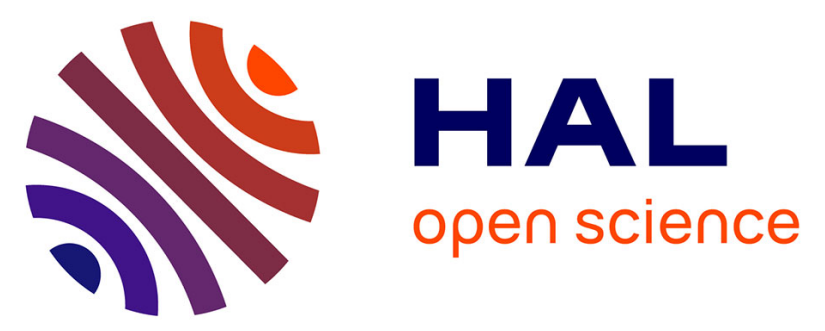

\title{
Empirical flow rate/pressure drop relationships for capillaries of triangular and rectangular cross-sections to be used in yield stress fluid porosimetry
}

Terence Emery Mackaya, Azita Ahmadi-Senichault, Abdelaziz Omari, Antonio Rodríguez de Castro

\section{To cite this version:}

Terence Emery Mackaya, Azita Ahmadi-Senichault, Abdelaziz Omari, Antonio Rodríguez de Castro. Empirical flow rate/pressure drop relationships for capillaries of triangular and rectangular crosssections to be used in yield stress fluid porosimetry. Transport in Porous Media, 2021, 136 (2), pp.587-605. 10.1007/s11242-020-01531-9 . hal-03307476

\section{HAL Id: hal-03307476 \\ https://hal.inrae.fr/hal-03307476}

Submitted on 26 Aug 2021

HAL is a multi-disciplinary open access archive for the deposit and dissemination of scientific research documents, whether they are published or not. The documents may come from teaching and research institutions in France or abroad, or from public or private research centers.
L'archive ouverte pluridisciplinaire HAL, est destinée au dépôt et à la diffusion de documents scientifiques de niveau recherche, publiés ou non, émanant des établissements d'enseignement et de recherche français ou étrangers, des laboratoires publics ou privés. 


\title{
Empirical Flow Rate/Pressure Drop Relationships for Capillaries of Triangular and Rectangular Cross-Sections to be Used in Yield Stress Fluid Porosimetry
}

\author{
Terence Emery Mackaya ${ }^{1}$ - Azita Ahmadi-Senichault ${ }^{1} \cdot$ Abdelaziz Omari $^{2}$. \\ Antonio Rodríguez de Castro ${ }^{3}$ (D)
}

\begin{abstract}
The aim of the present work is to investigate the flow rate/pressure gradient relationship for the flow of yield stress fluids through rectilinear capillaries of non-circular cross-sections. These capillaries very often serve as basic elements in the modeling of porous media as bundles of capillaries or pore-network models. Based on the notions of shape coefficient and critical Bingham number, empirical flow rate/pressure gradient relationships have been proposed for both Bingham and Herschel-Bulkley fluids. The reliability of these relationships has been assessed by performing numerical simulations with the open-source Computational Fluid Dynamics (CFD) package OpenFOAM. For the considered cross-sectional shapes (equilateral triangle and square), and for a wide range of Bingham numbers, the predictions of the proposed empirical relationships have shown to be in very good agreement with the results of the current numerical simulations, as well as with previous results from the literature. An interesting feature of the proposed empirical relationships is the possibility to easily predict the total flow rate under a given imposed pressure gradient in a bundle of non-circular capillaries having any random distribution of inscribed circle radii. Furthermore, in the context of the yield stress fluid porosimetry method (YSM), experimental data may now be processed based upon bundles of capillaries with non-circular cross-sections.
\end{abstract}

Keywords Yield stress fluids · Pore-size distribution · Non-circular cross-sections $\cdot$ Shape coefficient $\cdot$ Critical Bingham number

Antonio Rodríguez de Castro

antonio.rodriguezdecastro@ensam.eu

1 Arts et Metiers Institute of Technology, Université de Bordeaux, CNRS, INRA, INP, I2M, HESAM Université, 33400 Talence, France

2 Bordeaux INP, CNRS, I2M Bordeaux, Esplanade des Arts et Métiers, 33405 Talence Cedex, France

3 Arts et Metiers Institute of Technology, MSMP, HESAM Université, Rue Saint-Dominique, 51006 Châlons-en-Champagne, France 


\section{Introduction}

Porous media are encountered almost everywhere in everyday life, but also in several industrial applications, including oil recovery processes, subsurface solute transport, soil remediation and $\mathrm{CO}_{2}$ storage. In order to correctly describe the phenomena taking place in porous media (mass, momentum and heat transfer, particle trapping, etc.), an appropriate modeling of their internal structure is necessary. For this purpose, different characterization methods are available, among which the three-dimensional imaging techniques such as tomography or X-ray micro-computed tomography are of special relevance (Burlion et al. 2006; Wildenschild and Sheppard 2013; Houston et al. 2017). These techniques present the advantage of being non-destructive, providing reconstructions of the actual pore geometry with a resolution of $\sim 1 \mu \mathrm{m}$, and allowing the assessment of the topology of porous media (e.g., pore-size distribution, pores shapes, connectivity and tortuosity). However, the required equipment is costly and only a small volume of the porous medium is analyzed, questioning its representativeness.

Mercury intrusion porosimetry (MIP) technique is still commonly used because of its simplicity and feasibility (Peng et al. 2017). In MIP, mercury is forced into a sample of a porous medium under controlled conditions by incrementally increasing the applied pressure. The volume of mercury invading the sample is monitored, providing information on the microstructural features of the pore space by assuming a model geometry, due to the actual complexity of real pores. Frequently, the porous sample is idealized as a bundle of rectilinear capillaries of circular cross-section. Despite its rough simplicity, this capillary bundle model has proven to give a reasonably representative picture of the pore-size distributions (PSDs) of many real porous media. The major drawback of MIP is the toxicity of mercury vapors, the legally binding regulations and the legal prohibition in the coming years. Therefore, significant efforts have been made to find an alternative to conventional MIP. In particular, an innovative method based on the use of yield stress fluids was recently proposed, known as yield stress fluid porosimetry method (YSM). Yield stress fluids are viscoplastic materials which behave as solids under a critical shear stress and flow like liquids above this shear stress threshold. YSM was implemented by several authors (Malvault 2013; Rodríguez de Castro et al., 2014), based on the early work of Ambari et al. (1990). These authors showed that the PSD of the investigated medium can be extracted from the inversion of the raw experimental measurements of pressure gradient $\nabla P$ versus flow rate $Q$ performed at the steady state, provided that the yield stress fluid's characteristics are conveniently selected. The critical aspects and robustness of YSM were also assessed in the literature (Rodríguez de Castro et al. 2016, 2018).

Rodríguez de Castro et al. (2016) compared the PSD obtained by YSM with those obtained from MIP for a set of highly permeable sandstones and synthetic filters. They observed that even if comparable PSDs are provided by both methods, the results do not rigorously coincide. In a recent article, Rodríguez de Castro et al. (2018) discussed the impact of the shear rheology parameters of yield stress fluids on the accuracy of the PSDs obtained with YSM. Also, in order to explore the influence of the irregular form of pore cross-section and its spatial variation, Malvault et al. (2017) performed numerical simulations of Bingham fluids flowing in a bundle of straight capillaries of non-circular crosssection shape with varying characteristic dimension along the capillary axis. They showed that the calculated $Q(\nabla P)$ approached more closely the experimental data than the traditional model of circular capillaries with constant cross-section. However, the required computational cost was high due to the 3D nature of the problem. In this respect, Kefayati and 
Huilgol (2017) proposed a lattice Boltzmann method for the simulation of the steady flow of Bingham fluids in a pipe of square cross-section, leading to a considerable reduction of the computation time.

In order to avoid costly and/or complex numerical simulations, one may ask whether there exists a simple alternative to estimate flow rate/pressure gradient relationships without any loss of calculation accuracy. The goal of the present work is to contribute to this progress by focusing on the formulation of empirical flow rate/pressure gradient relationships for various types of yield stress fluids flowing through straight capillaries of noncircular cross-sections, particularly of square and equilateral triangular shapes. Prior to this and as a starting point, some basic literature results concerning the flow of Newtonian and Bingham fluids through rectilinear capillaries of circular and square cross-sections are recalled in the next section. On the basis of these results, empirical flow rate/pressure gradient relationships are subsequently presented for the general case of Herschel-Bulkley fluids flowing in capillaries of different cross-sections. Furthermore, numerical simulations are also performed in order to assess the reliability of the proposed relationships. Finally, two examples of their application in the context of YSM are presented.

\section{Literature Review}

\subsection{Newtonian Fluids Flow in Rectilinear Capillaries}

Over the past, the flow of Newtonian fluids through rectilinear capillaries of various crosssections has been extensively studied. Patzek and Silin (2001) reconsidered such a flow for capillaries with rectangular cross-section of height $2 \mathrm{a}$ and width $2 \mathrm{~b}$ by solving the momentum equation under creeping and non-slip conditions. These authors obtained the following analytical expression of the velocity profile

$$
U_{x_{3}}\left(x_{1}, x_{2}\right)=\frac{\nabla P}{2 \mu}\left[a^{2}-x_{2}^{2}+\frac{32 a^{2}}{\pi^{3}} \sum_{j=0}^{\infty} \frac{(-1)^{j+1} \cosh \left(\frac{a_{j} x_{1}}{a}\right) \cos \left(\frac{a_{j} x_{2}}{a}\right)}{(2 j+1)^{3} \cosh \left(\varepsilon a_{j}\right)}\right]
$$

where $a_{j}=\left(\frac{\pi}{2}\right)(2 j+1), \mu$ is the fluid dynamic shear viscosity of the fluid, and $\varepsilon=\frac{a}{b}$ is the aspect ratio $(a \leq b) . x_{1}$ and $x_{2}$ are the axis in the cross-section, and $x_{3}$ is the axis perpendicular to the cross-section, i.e., collinear to the velocity vector at each point. The flow rate versus pressure gradient $Q(\nabla P)$ relationship can then be easily obtained after the integration of Eq. (1) over the cross-section. In the particular case of a square cross-section $(\varepsilon=1)$, the mean velocity is given by:

$$
\bar{U}=\frac{Q}{S_{\text {pipe }}}=C_{S} \frac{a^{2} \nabla P}{8 \mu} \quad \text { with } \quad C_{S}=\left[\frac{8}{3}-16 \sum_{j=0}^{\infty} \frac{\tanh \left(a_{j}\right)}{a_{j}^{5}}\right]
$$

where $S_{\text {pipe }}=4 a^{2}$ is the cross-sectional area.

When $C_{S}=1$, Eq. (2) reduces to the Hagen-Poiseuille formula for a circular crosssection. Therefore, the shape coefficient $C_{S}$, is defined as the ratio between the volumetric flow rate of a Newtonian fluid corresponding to any cross-sectional shape and the one corresponding to a circular section with identical cross-sectional area $S_{\text {pipe }}$. In 
this regard, Patzek and Silin (2001) have determined the shape coefficient for various cross-section geometries. For equilateral triangular cross-sections, the obtained shape coefficient is $C_{T}=\frac{6}{5}$.

\subsection{Yield Stress Fluids Flow in Straight Cylindrical Capillaries}

The Herschel-Bulkley empirical relationship (Herschel and Bulkley 1926) is one of the most widely used viscoplastic models:

$$
\begin{cases}\underline{\dot{\gamma}}=0 & \text { for } \quad \tau \leq \tau_{0} \\ \underline{\underline{\tau}}=\left(\frac{\tau_{0}}{\dot{\gamma}}+k \cdot \dot{\gamma}^{n-1}\right) \underline{\underline{\gamma}} & \text { for } \quad \tau \geq \tau_{0}\end{cases}
$$

where $\underline{\underline{\tau}}$ and $\underline{\underline{\gamma}}$ are, respectively, the shear stress and the strain rate tensors. $\tau_{0}$ is the yield stress of the fluid, $k$ is consistency, and $n$ is fluidity index. The particular case $n=1$ corresponds to the ideal Bingham plastic model. In the preceding equation, the strain rate tensor is defined by $\underline{\underline{\dot{\gamma}}}=\left[\nabla \overrightarrow{\boldsymbol{U}}+(\nabla \overrightarrow{\boldsymbol{U}})^{T}\right]$, where $\overrightarrow{\boldsymbol{U}}$ is the velocity vector and the superscript $T$ denotes the transpose. The magnitudes of the two tensors $\underline{\tau}$ and $\dot{\gamma}$ are denoted $\tau$ and $\dot{\gamma}$, respectively, and can be obtained as $\tau=\sqrt{\frac{1}{2} I I_{\underline{\tau}}}=\sqrt{\left(\frac{1}{2}\right)(\underline{\underline{\tau}}: \underline{\underline{\tau}})} \quad$ and $\underline{\underline{\tau}}=\left[k \dot{\gamma}^{n-1}+\frac{\tau_{0}[1-\exp (-m \dot{\gamma})]}{\dot{\gamma}}\right] \underline{\underline{\dot{\gamma}}}$, where the symbol $I I$ is the second invariant of the considered tensor.

\subsubsection{Flow Structure in Capillaries of Arbitrary Cross-Section}

Mosolov and Miasnikov (1965, 1966, 1967) analyzed, from a theoretical point of view, the creeping steady-state flow of yield stress fluids through rectilinear capillaries of arbitrary cross-sections. By analytically solving an energy balance equation for an imposed pressure gradient, these authors proved that the curvature of the flow region is everywhere greater than a threshold value $l^{*}$, that only depends on the shape of the cross-section. Consequently, the flow under a pressure gradient $\nabla P$, of a fluid with a yield stress $\tau_{0}$ through a capillary of a given cross-section can take place only if the following condition is met:

$$
\frac{\tau_{0}}{l^{*}} \leq \nabla P
$$

In general, the flow structure consists of a central plugged region where the fluid is unsheared, surrounded by a sheared region. Also, when the cross-sectional shape presents angular corners, there exists a dead zone in contact with the solid walls where the fluid is at rest. On the basis of these theoretical works, Huilgol (2006) analytically investigated the flow of different types of yield stress fluids (Bingham, Casson, Herschel-Bulkley), through rectilinear capillaries having at least one symmetry axis. This 
author concluded that $l^{*}$ does not depend on the considered type of yield stress fluid, but only on the cross-section shape of the capillary through which the fluid is flowing.

\subsubsection{Numerical Computation of Flow Rate for Bingham Fluids}

The flow of Bingham fluids through rectilinear capillaries of square cross-section under creeping flow conditions was numerically studied by Saramito and Roquet (2001). For such fluids, the occurrence of the flow and the relative extent of each zone is controlled by the value of the non-dimensional Bingham number, $B_{i}=\tau_{0} / \sigma_{0}$, which quantifies the importance of yield stress $\tau_{0}$ as compared to the excess shear stress $\sigma_{0}$ produced in the constant plastic viscosity region. In their study, Saramito and Roquet (2001) used $\sigma_{0}=\frac{a \nabla P}{2}$, where $a$ is the radius of the circle inscribed in the square cross-section of the capillary. They found that the dimensionless mean velocity $\bar{U} / U^{*}$ may be expressed as:

$$
\frac{\bar{U}}{U^{*}}=\frac{C_{S}}{4}\left[1-\frac{4}{3} \frac{B_{i}}{B_{i c}}+\frac{1}{3}\left(\frac{B_{i}}{B_{i c}}\right)^{4}\right]
$$

with $U^{*}$ being a characteristic velocity defined as:

$$
U^{*}=\frac{a^{2} \nabla P}{2 k}=\frac{a \sigma_{0}}{k}
$$

$C_{s}$ is the shape coefficient that equals unity for a circular cross-section. The shape coefficient accounts for the deviation of the actual cross-section shape from circularity and is the same as in Eq. (2), since it depends only on the shape of the considered cross-section. $B_{i c}$ is the critical Bingham number above which the fluid cannot flow. When $B_{i}$ increases, the contour of the central plugged zone moves toward solid walls and the dead zones become larger, leading to a thinner sheared region in between. At $B_{i}=B_{i c}$, the sheared zone between the central plugged zone and the dead ones disappears, and the flow stops. Therefore, the onset of flow condition given by Eq. (4) may be rewritten as $\frac{B_{i}}{B_{i c}}<1$ with $B_{i c}=\frac{2 l^{*}}{a}$. For a square cross-section (with $2 a$ being the side length), the characteristic length is $l^{*}=\frac{2 a}{2+\sqrt{\pi}}($ Mosolov and Miasnikov 1965, 1966, 1967). Consequently, the critical Bingham number for the square cross-section can be expressed as:

$$
B_{i c}^{S}=\frac{4}{2+\sqrt{\pi}}
$$

On the basis of detailed numerical simulations of Bingham fluids' flow using an adaptive finite element method, Saramito and Roquet (2001) obtained the value $B_{i c}^{S}=1.07$, while the calculation of Damianou and Georgiou (Damianou and Georgiou 2014) gave a value of 1.06, which is closer to the theoretical value 1.06038 given by Eq. (7). Recently, Malvault et al. (2017) carried out numerical simulations under similar conditions finding $B_{i c}=1.0416$, which also agrees acceptably with the theoretical value (error less than $2 \%$ ). 


\section{Proposed Empirical Q $(\nabla P)$ Relationships}

It is recalled that the objective of the present work is to show that simple empirical relationships may be used to quantitatively predict $Q(\nabla P)$ for the flow of yield stress fluids in rectilinear capillaries of various cross-section shapes, with only marginal loss of accuracy compared to numerical simulations. To do so, the empirically calculated $Q(\nabla P)$ will systematically be compared to the simulation results already published in previous works and/or to the ones computed in the current study.

From the literature review presented in the previous sections, it seems that whatever the fluid and the cross-section shape, the dimensionless mean velocity in steady-state creeping conditions may be factorized and expressed as a product of a shape coefficient and a flow term involving $B_{i}$ and $B_{i c}$. In the case of Bingham fluids, the starting point is Eq. (5) proposed by Saramito and Roquet (2001) for a square cross-section. In order to extend it to the case of equilateral triangular cross-sections, $B i_{\mathrm{c}}$ will be rewritten as proposed by Mosolov and Miasnikov (Mosolov and Miasnikov 1965, 1966, 1967):

$$
B_{i c}^{T}=\frac{6}{3+\sqrt{\pi \sqrt{3}}}
$$

Then, based on a similar hydraulic analogy previously used by Saramito and Roquet (2001), it is proposed that the normalized mean velocity for Bingham fluids flowing through rectilinear capillaries of equilateral triangular cross-section can be expressed as:

$$
\frac{\bar{U}}{U^{*}}=\frac{C_{T}}{4}\left[1-\frac{4}{3} \frac{B_{i}}{B_{i c}^{T}}+\frac{1}{3}\left(\frac{B_{i}}{B_{i c}^{T}}\right)^{4}\right]
$$

with $U^{*}$ being a characteristic velocity previously defined in Eq. (6) and $\bar{U}$ being the mean flow velocity, i.e., $\bar{U}=\frac{Q}{S_{\text {pipe }}}$.

In order to obtain analogous expressions for the more complex case of Herschel-Bulkley fluids, it is first recalled that the characteristic curvature $l^{*}$ is independent of the type of yield stress fluid (Huilgol 2006) and Eq. (4), which connects the pressure gradient and $\tau_{0}$, is insensitive to the exact rheology of the considered yield stress. Therefore, the critical Bingham number $B_{i c}=2 l^{*} / a$ is also independent of the rheological model, and Eqs. (7) and (8) can be used for cross-sections of square and equilateral triangle shapes, respectively. Besides, based on the previous definitions of $B_{i}$ and $U^{*}$, as well as the well-known $Q(\nabla P)$ relationships of Herschel-Bulkley fluids flowing through a straight capillary of circular cross-section, the normalized velocity $\bar{U} / U^{*}$ can be written as:

$$
\frac{\bar{U}}{U^{*}}=\left(1-B_{i}\right)^{\frac{1}{n}+1}\left[\frac{\left(1-B_{i}\right)^{2}}{3+\frac{1}{n}}+\frac{2 B_{i}\left(1-B_{i}\right)}{3+\frac{1}{n}}+\frac{B_{i}^{2}}{1+\frac{1}{n}}\right]
$$

where the characteristic flow velocity $U^{*}$ and the Bingham number are, respectively, given by: 


$$
\begin{gathered}
U^{*}=\frac{a\left(\frac{a \nabla P}{2}\right)^{\frac{1}{n}}}{k^{\frac{1}{n}}} \\
B_{i}=\frac{\tau_{0} a^{n}}{k U^{* n}}
\end{gathered}
$$

Then, as previously done by Saramito and Roquet (2001) for Bingham fluids, Eq. (10) is now extended to the cases of Herschel-Bulkley fluids for square and triangular crosssections as:

$$
\frac{\bar{U}}{U^{*}}=C\left(1-\frac{B_{i}}{B_{i c}}\right)^{\frac{1}{n}+1}\left[\frac{\left(1-\frac{B_{i}}{B_{i c}}\right)^{2}}{3+\frac{1}{n}}+\frac{2 \frac{B_{i}}{B_{i c}}\left(1-\frac{B_{i}}{B_{i c}}\right)}{3+\frac{1}{n}}+\frac{\left(\frac{B_{i}}{B_{i c}}\right)^{2}}{1+\frac{1}{n}}\right]
$$

where $C$ is the shape coefficient of the considered cross-section shape, i.e., $C_{T}=6 / 5$ for an equilateral triangle and $C_{S}$ for a square (Eq. 2). $B_{i c}$ is the corresponding critical Bingham number given by Eqs. (7) and (8) for square and triangular cross-sections, respectively. It is worth mentioning that Eqs. (13) and (10) are identical for a circular cross-section with $C=1$ and $B_{i c}=1$. Moreover, for the case of a square cross-section, Eq. (13) reduces to Eq. (5) earlier proposed by Saramito and Roquet (2001) for a Bingham fluid (with fluidity index $n$ equal to 1$)$.

\section{Assessment of the Empirical Expressions by Means of Numerical Simulations}

In order to assess the reliability of the proposed empirical flow rate/pressure gradient relationships, the obtained predictions are hereafter compared with simulation results. These simulation results come from the literature (Malvault et al. 2017) in the case of Bingham fluids or were specifically performed in the current work for Herschel-Bulkley fluids.

\subsection{Governing Equations and CFD Tool}

The steady-state, isothermal, incompressible and creeping flow of yield stress fluids through rectilinear capillaries with a square or equilateral triangle cross-sectional shape is considered. For all the investigated geometries, a no-slip boundary condition $(\overrightarrow{\boldsymbol{U}}=\overrightarrow{0})$ is set at the solid wall of the capillary. A uniform and constant pressure gradient is imposed between the inlet and the outlet $\left(P_{\text {inlet }}=P_{i}\right.$ and $\left.P_{\text {outlet }}=0 \mathrm{~Pa}\right)$. Moreover, the zero gradient boundary condition is chosen for both the inlet and the outlet $(\nabla \overrightarrow{\boldsymbol{U}} \cdot \overrightarrow{\boldsymbol{n}})$, where $\overrightarrow{\mathbf{n}}$ is the unitary vector pointing outside of the domain. The flow is governed by the momentum and the continuity equations, which reduce to the following expressions when body forces are neglected:

$$
\left\{\begin{array}{l}
\nabla \cdot \underline{\underline{\tau}}-\nabla P=0 \\
\nabla \cdot \bar{U}=0
\end{array}\right.
$$


where the stress tensor $\underline{\underline{\tau}}$ is given by Eq. (3), $P$ is the absolute pressure at the local scale and $\boldsymbol{U}$ is the velocity vector. Moreover, the Reynolds number is here defined as:

$$
R_{e}=\frac{\rho \bar{U}^{2-n}(2 a)^{n}}{k}
$$

where $\rho$ is the density of the fluid. $R_{e}$ must be significantly lower than unity to ensure creeping flow conditions.

\subsection{Case of Bingham Fluids}

Before dealing with the case of Herschel-Bulkley fluids, the case of a Bingham fluids is first considered. The flow of such fluids in rectilinear capillaries of square cross-section was extensively discussed in several previous works (Saramito and Roquet 2001; Roquet and Saramito 2008; Damianou et al. 2016). Therefore, to evaluate the results provided by the present empirical relationship (Eq. 10), only the case of the equilateral triangular crosssection is considered in this study, using simulation data available in the literature for comparison purposes. Figure 1 shows the flow rate normalized by the quantity $q_{c}=a^{3}\left(\frac{\tau_{0}}{k}\right)^{\frac{1}{n}}$, versus dimensionless pressure gradient of Malvault et al. (2017) together with those obtained by using Eq. (10). As it can be observed, there is a very good agreement between numerical results and predictions over a wide range of $B_{i}$ values.

\subsection{Case of Herschel-Bulkley Fluids}

In the second assessment step, the flow of Herschel-Bulkley fluids through capillaries of square and equilateral triangular cross-sections is considered. For this case, the governing Eq. (14) is solved using OpenFOAM, which is an open-source multi-physics code based on a finite volume discretization. With the SimpleFoam solver, the coupling between the

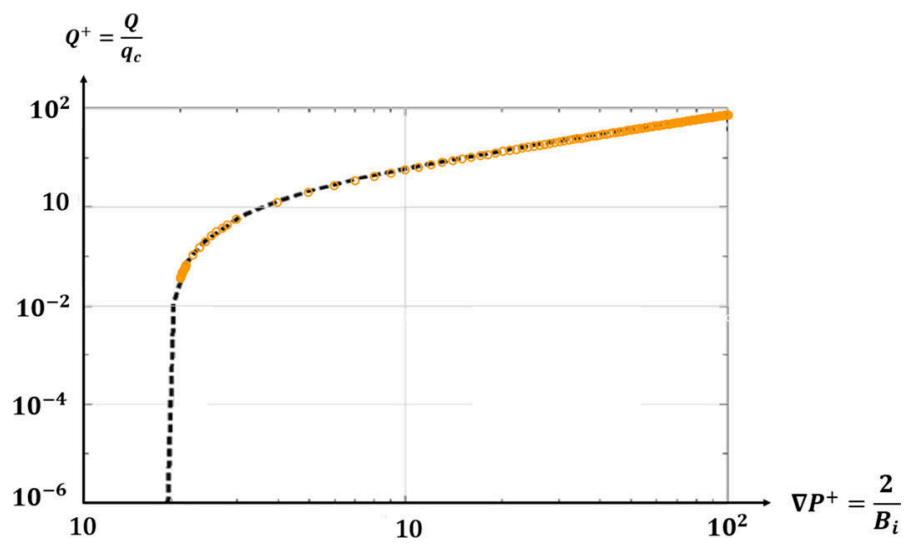

Fig. 1 Dimensionless flow rate $Q^{+}$versus dimensionless pressure gradient for a Bingham fluid $\left(\tau_{0}=1 \mathrm{~Pa}, n=1, k=1 \mathrm{~Pa} . \mathrm{s}\right)$ flowing through a capillary of equilateral triangular cross-section. Black dashed line: simulation results of Malvault et al. (2017). Orange points: prediction obtained by Eq. (9) 
velocity and pressure is ensured thanks to the iterative algorithm SIMPLE (Semi-Implicit Method for Pressure Linked Equations). The velocity system is solved using the "Smoothsolver" linear solver, with the Gauss-Seidel smoother. The pressure is solved using the geometric algebraic multigrid (GAMG) linear solver, associated to the Gauss-Seidel smoother. In the present investigation, it is assumed that the shear rheology of yield stress fluids is well described by the Herschel-Bulkley model (Eq. 3). However, a continuous model is required in numerical experiments to avoid singularities. In the present work, a regularized model similar to the one proposed by Papanastasiou (1987) is used, in which the stress tensor is expressed as:

$$
\underline{\underline{\tau}}=\left[k \dot{\gamma}^{n-1}+\frac{\tau_{0}[1-\exp (-m \dot{\gamma})]}{\dot{\gamma}}\right] \underline{\underline{\gamma}}
$$

where $m$ is the regularization parameter. Moreover, $\max \left(\dot{\gamma}, \dot{\gamma}_{\min }\right)$ is used instead of $\dot{\gamma}$, where $\dot{\gamma}_{\min }$ is a user-defined minimum value for the shear rate used to avoid any divergence. For accuracy purposes, the value $\dot{\gamma}_{\min }=2 \times 10^{-14} \mathrm{~s}^{-1}$ has been chosen in the present simulations.

The value of $m$ determines the range of shear rates over which the Herschel-Bulkley model (Eq. 3) is accurately approximated by the regularized model (Eq. 16) and it depends on the set of parameters $\left(\tau_{0}, k, n\right)$. Therefore, depending on the range of flow rates in the capillary (or the range of shear rates), different values of $m$ can be adequate to mimic the rheological behavior of the fluid.

It is worth noting that, inherently, the regularized model assumes that all fluid regions are sheared, so no plugged regions exist strictly speaking (Zhu et al. 2005). Instead, the fluid exhibits a very high viscosity at very low shear rates and pseudoplastic behavior at high shear rates. Several authors (e.g., Damianou et al. 2014; Panaseti and Georgiou 2017) reported that the value of the parameter $m$ plays an important role in the accuracy and the location of the yield surfaces defined by the set of points for which $\tau=\tau_{0}$. In addition, some authors pointed out that the greater the value of the Bingham number, the greater is the value of $m$ that ensures high accuracy of the topography of the yield surfaces (Damianou and Georgiou 2014).

In the present study, and for the case of a square cross-section, a systematic investigation was carried out to determine the value of $m$ that ensures sufficient accuracy. That is, for each Bingham number, different simulations were conducted with increasing values of $m$ until the criteria on the normalized residuals were met (less than $10^{-7}$ for both the velocity and pressure fields), and the relative variation of the flow rate over the last 1000 iterations was less than $0.004 \%$. Then, at a given Bingham number, the last value of $m$ was selected when the relative error on the converged volumetric flow rate (compared to the one obtained with the previous value of $m$ ) was less than $0.004 \%$. After this assessment, for each Bingham number, the retained value of $m$ was subsequently used for the simulations with equilateral triangular cross-sections. It should be highlighted that, the objective of this study being to numerically assess the validity of the empirical flow rate/pressure gradient relationships, the selected value of $m$ has been chosen so that it provides stabilized simulated flow rates while keeping reasonable simulation time. It is well known that for a given pressure gradient and when $Q$ approaches its asymptotic value a significant change of $\mathrm{m}$ has only a very small impact on velocity magnitude but can still affect the location of envelopes of unsheared fluid (Damianou and Georgiou 2014). 


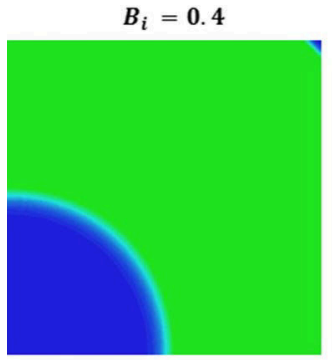

$m=55.10^{3} \mathrm{~s}$
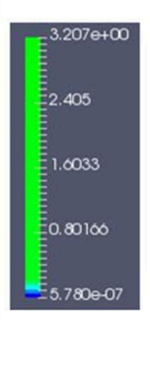

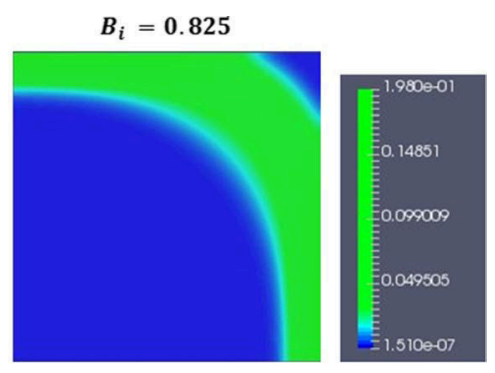

$m=90.10^{3} \mathrm{~s}$

Fig. 2 Magnitude of the strain rate tensor (in $\mathrm{s}^{-1}$ ) for the flow of a Herschel-Bulkley fluid $\left(\tau_{0}=1 \mathrm{~Pa}, n=0.6\right.$ and $\left.k=1 \mathrm{~Pa} \mathrm{~s}^{0.6}\right)$ in a capillary of square cross-section, for two values of $B i$. Different colors correspond to different magnitudes of the strain rate tensor, with greenish colors corresponding to higher values and bluish colors corresponding to nearly stagnant fluid

Table 1 Comparison between empirical and simulated dimensionless flow rate of a Herschel-Bulkley fluid through a capillary of square cross-section, for different values of $B_{i}$

\begin{tabular}{lllllll}
\hline$B_{i}$ & 0.25 & 0.4 & 0.6 & 0.725 & 0.825 & 0.9 \\
\hline$m(\mathrm{~s})$ & $40 \times 10^{3}$ & $55 \times 10^{3}$ & $70 \times 10^{3}$ & $80 \times 10^{3}$ & $90 \times 10^{3}$ & $10^{5}$ \\
$Q_{\text {empirical }}^{+}$ & 5.4072 & 1.5503 & 0.3353 & 0.1123 & 0.0371 & 0.012 \\
$Q_{\text {simulated }}^{+}$ & 5.415 & 1.5528 & 0.3358 & 0.1125 & 0.0372 & 0.01203 \\
Relative errors (\%) & 0.144 & 0.161 & 0.149 & 0.17 & 0.296 & 0.25 \\
\hline
\end{tabular}

Concerning the meshing of the geometries, all the considered computational domains were 3D. Hence, in order to reduce the computational time, and for symmetry reasons, only a part of the whole domain was modeled. Moreover, since the solution of the problem does not change along the axial direction, only a limited number of cells are needed in that direction. Consequently, mesh refinement was made only in the transverse directions by increasing the number of cells. The refinement was such that the relative variation of the computed volumetric flow rate between two successive mesh sizes were less than $0.5 \%$ for triangular cross-sections and less than $0.1 \%$ for square cross-sections. Furthermore, since for square and equilateral triangular cross-sections the values of the critical Bingham numbers are, respectively, 1.0603 (Eq. 7) and 1.1251 (Eq. 8), the highest value of Bi considered was 0.9 .

The volumetric flow rate values obtained through the numerical simulations were then compared with those predicted by the approximate flow rate/pressure gradient relationship given by Eq. (13). For that purpose, all the volumetric flow rates were normalized by the quantity $q_{c}=a^{3}\left(\frac{\tau_{0}}{k}\right)^{\frac{1}{n}}$. Finally, independently of the cross-section shape, the chosen values of the Bingham numbers were so that the corresponding Reynolds numbers span the range $0.1>R_{e}>10^{-7}$, ensuring creeping flow conditions.

\subsubsection{Simulation Results}

The flow structure for the considered Herschel-Bulkley fluid $\left(\tau_{0}=1 \mathrm{~Pa}, n=0.6\right.$ and $k=1 \mathrm{~Pa} . \mathrm{s}^{0.6}$ ) flowing through a capillary of square cross-section 
Table 2 Comparison between empirical and simulated dimensionless flow rate of a Herschel-Bulkley fluid through a capillary of triangular cross-section, for different values of $B_{i}$

\begin{tabular}{lllllll}
\hline$B_{i}$ & 0.25 & 0.4 & 0.6 & 0.725 & 0.825 & 0.9 \\
\hline$m(\mathrm{~s})$ & $40 \times 10^{3}$ & $55 \times 10^{3}$ & $70 \times 10^{3}$ & $80 \times 10^{3}$ & $90 \times 10^{3}$ & $10^{5}$ \\
$Q_{\text {empirical }}^{+}$ & 7.7957 & 2.326 & 0.5535 & 0.2082 & 0.082 & 0.0342 \\
$Q_{\text {simulated }}^{+}$ & 7.852 & 2.342 & 0.5574 & 0.2097 & 0.08287 & 0.0346 \\
Relative errors (\%) & 0.722 & 0.688 & 0.705 & 0.72 & 1.061 & 1.17 \\
\hline
\end{tabular}

is displayed in Fig. 2 for two distinct values of $\mathrm{Bi}$. The corresponding values of $m$ that are determined following the procedure described in the preceding paragraph are also provided. It can be deduced that the change of the flow structure is usual: an inner plugged zone (with very low shear rates) surrounded by a sheared zone and stagnant fluid regions at the corners (Damianou et al. 2014; Damianou and Georgiou 2014). As Bi is increased (the pressure gradient decreased), the velocity decreases in the rigid zone that grow in size while simultaneously the stagnant zone expand and the sheared zone between the two become thinner. The rate of expansion of the central zone is greater than that of the dead zone and comes in contact at $B_{i}=B_{i c}$ when the capillary is fully plugged.

Concerning the variation of the non-dimensional flow rate with respect to $\mathrm{Bi}$, Tables 1 and 2 provide comparisons between the simulation results, $Q_{\text {simulated }}^{+}$, and the predictions obtained from Eq. (13), $Q_{\text {empirical }}^{+}$, for the square and equilateral triangle cross-sections, respectively. The corresponding values of $m$ are also listed. As it can be noticed, $Q_{\text {empirical }}^{+}$and $Q_{\text {simulated }}^{+}$are very close for all the considered Bingham numbers, with relative errors that are lower than $\sim 1 \%$. Therefore, it can be put forward that the proposed dependence of normalized mean velocity $\bar{U} / U^{*}$ on the ratio $B_{i} / B_{i c}$ provides acceptable predictions over the considered range of $B_{i}$ values under creeping flow conditions. It is worth mentioning that the relative errors between the simulation and the empirical approaches depend on the value of $\mathrm{Bi}$ for all the considered cases. This is related to the accuracy of the simulated flow structure due to somewhat coarse sampling when screening $\mathrm{m}$ values.

\section{Application to YSM}

As mentioned in the introduction, the yield stress fluids porosimetry method (YSM) has been based until now on modeling the porous medium as being a bundle of capillaries of circular cross-sections. In this section, the empirical flow rate/pressure gradient relationships developed in the current study are used to investigate the flow of yield stress fluids in bundles of capillaries with either square or equilateral triangle cross-sectional shapes. This is expected to improve the representativeness of the PSDs obtained with YSM.

It is interesting to highlight the fact that modeling a porous sample of a given porosity as a bundle of capillaries with circular, square or triangular cross-sections has a significant impact on the specific surface areas involved. Furthermore, the pore-surfaceto-pore-volume ratio, which is directly proportional to the specific surface area for a given porosity, is different for rectilinear capillaries of equal cross-sectional surface 
Fig. 3 Schematic representation of capillaries with various cross-sections having the same radius, $a$, of inscribed circle. For an arbitrary flow magnitude, the central plug and dead regions are represented in blue
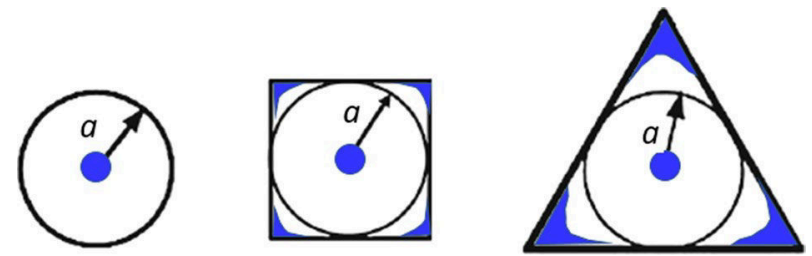

but dissimilar in shape. In the particular cases of circular, square and triangular crosssections, the pore-surface-to-pore-volume ratios are $2 \sqrt{\pi}, 4$ and $6 / \sqrt[4]{3}$, respectively. Therefore, given that it is well known that the specific area is a key parameter in surface-dependent phenomena such as adsorption, the relevance of the PSDs provided by YSM can be questioned when only circular capillaries are considered.

Hereafter, a hypothetical cylindrical porous sample of diameter $2 R=5 \mathrm{~cm}$ and arbitrary length, with porosity $\emptyset=0.4$, is considered. It is assumed that the pores can be represented as a bundle of rectilinear capillaries with alternatively circular, square, or equilateral triangular cross-sections. Moreover, the three types of model porous media are assumed to have a bimodal distribution $p(a)$ of the radii of the inscribed circles in the considered cross-section (as displayed in Fig. 3) and given as follows:

$$
p(a)=\left[\frac{w_{1}}{\sigma_{1} \sqrt{2 \pi}} \exp \left(-\frac{\left(a-m_{1}\right)^{2}}{2 \sigma_{1}^{2}}\right)+\frac{w_{2}}{\sigma_{2} \sqrt{2 \pi}} \exp \left(-\frac{\left(a-m_{2}\right)^{2}}{2 \sigma_{2}^{2}}\right)\right]
$$

where $w_{1}=\frac{2}{3}, w_{2}=\frac{1}{3}, m_{1}=12 \mu \mathrm{m}, m_{2}=24 \mu \mathrm{m}, \sigma_{1}=3 \mu \mathrm{m}$ and $\sigma_{2}=6 \mu \mathrm{m}$.

By keeping the porosity constant, the number of capillaries forming each bundle is calculated as follows:

$$
N_{\text {cap }}=\beta \frac{\pi \emptyset R^{2}}{\int_{a_{\min }}^{a_{\max }} p(a) \pi a^{2} \mathrm{~d} a}
$$

with $\beta=1$ for the circular cross-section, $\beta=\frac{\pi}{4}$ for the square one and $\beta=\frac{\pi}{3 \sqrt{3}}$ for the triangular one. The term in the numerator of Eq. (18) represents, apart from the constant $\beta$, the total sum of the cross-sectional areas of all capillaries composing the bundle.

First of all, a numerically designed experiment is considered. Our Herschel-Bulkley fluid with $\tau_{0}=10 \mathrm{~Pa}, n=0.6$ and $k=1$ Pa.s.6 is injected through the three bundles of capillaries, by imposing 50 predetermined pressure gradients. We choose 50 equidistant pore radius classes $a_{i}$ in the range between $a_{\min }=m_{1}-3 \sigma_{1}$ and $a_{\max }=m_{2}+3 \sigma_{2}$. These extreme values allow the calculation of $\nabla P_{\min }=2 \tau_{0} / a_{\max }$ and $\nabla P_{\max }=2 \tau_{0} / a_{\min }$, thus defining the range of pressure gradients to be applied through the different model porous samples. The intermediate pressure gradients are given by $\nabla P_{i}=2 \tau_{0} / a_{i}$. Since the flow rate pressure gradient relationship is known for a Herschel-Bulkley fluid flow through a single capillary, it is now possible to compute the total flow rate through each bundle for any imposed pressure gradient as follows:

$$
Q(\nabla P)=N_{\text {cap }} \int_{\frac{2 \tau_{0}}{B_{i c} \mathrm{P}}}^{a_{\max }} q(\nabla P, a) p(a) \mathrm{d} a
$$



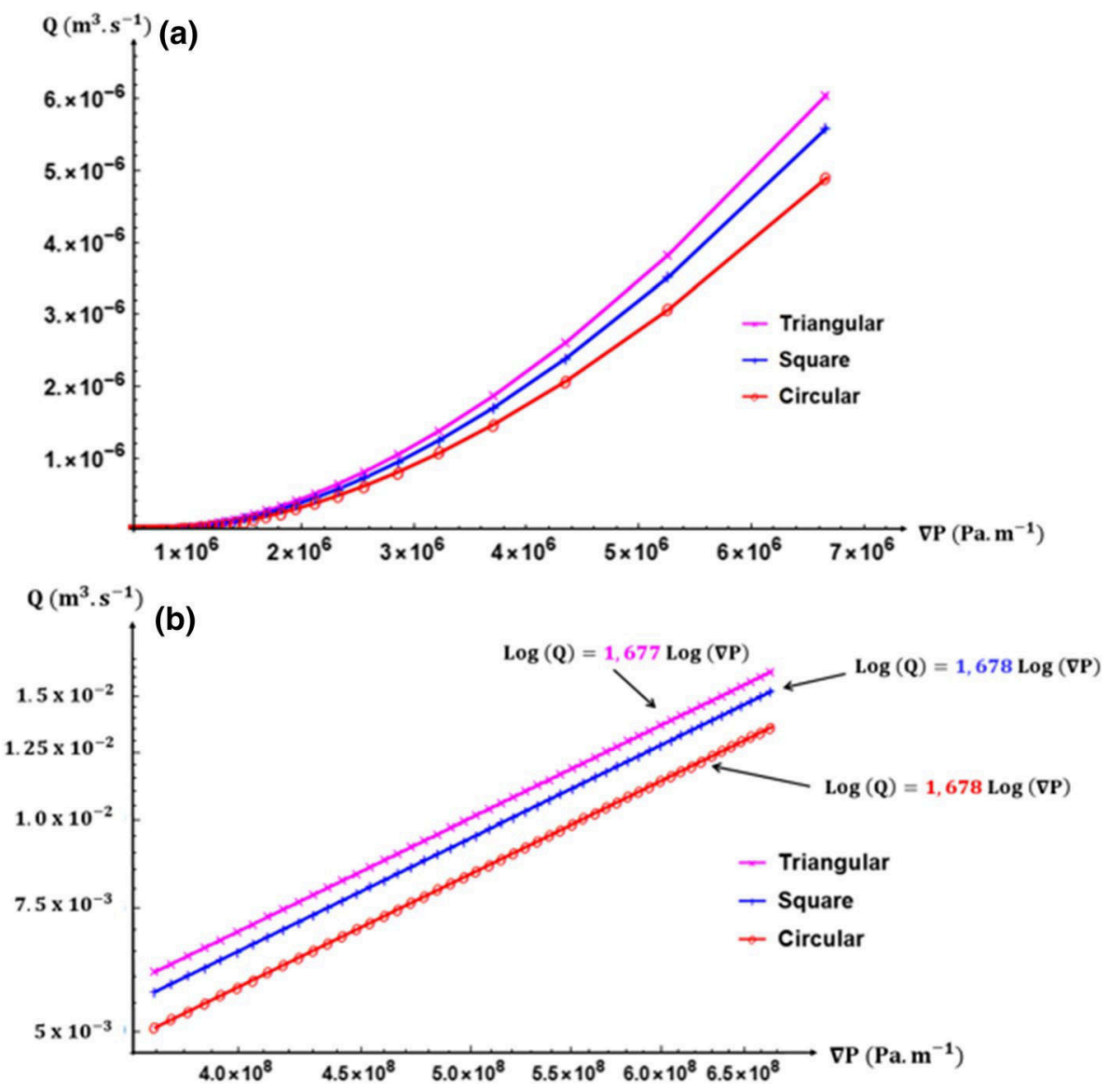

Fig. 4 a Characteristic curves for the flow of a Herschel-Bulkley fluid $\left(\tau_{0}=10 \mathrm{~Pa}, n=0.6\right.$ and $\left.k=1 \mathrm{~Pa}^{0.6}\right)$. b Zoomed image showing the trend of the three characteristic curves at high values of the imposed pressure gradients $\left(\nabla P_{A}=6.66 \times(10)^{7} \mathrm{~Pa} \mathrm{~m}^{-1}\right.$ and $\left.\nabla P_{A}=6.66 \times(10)^{8} \mathrm{~Pa} \mathrm{~m}^{-1}\right)$

where $q(\nabla P, a)$ is the flow rate through a single capillary with and inscribed circle of radius a.

The lower bound of the integral in Eq. (19) corresponds to the critical value of the inscribed circle below which there is no flow in the capillary for the considered $\nabla P$. The resulting flow rate/pressure gradient curves, also called "characteristic curves," are presented in Fig. 4a. This figure shows that for identical porosity, the cross-section shape of the capillaries has a significant impact on the obtained characteristic curve.

The difference from one characteristic curve to another is due to the form of the cross-section of the capillaries composing the bundles that have the same distribution of inscribed circles (Fig. 4). Therefore, at a given pressure gradient, the flow rate through capillaries of triangular cross-sections is greater than the one through capillaries of square cross-sections, which is greater, in turn, than the one through capillaries of circular crosssections. Moreover, Fig. 4 shows that the differences between the three curves increase as the pressure gradient increases. This is due to the fact that both plugged and dead zones (in 
blue in Fig. 4) become smaller as the value of the pressure gradient is increased. Therefore, not only more capillaries will participate in the flow, but also the regions of the capillary where the fluid actually flows for each pore size become larger for non-circular cross-sections (Fig. 4). In other respects, instead of comparing cross-sections that have the same inscribed circle one may compare cross-sections of same area and having different forms. In that case calculated flow rates at a given pressure gradient are ordered as circular $>$ square $>$ triangular provided that each of them is conducting. Such a hierarchy is linked to the extent of dead zones that are absent in the circular case and larger in case of triangular cross-sections.

In addition, the trend of these characteristic curves at $\nabla P>>\frac{2 \tau_{0}}{a_{\min }}$ also contains valuable information. Within this regime, and for a bundle of capillaries of circular cross-sections, it is known that the flow rate $Q(\nabla P)$ is expected to scale as $\nabla P^{\frac{1}{n}}$ (Rodríguez de Castro et al. 2014). Figure 4(b) is a log-log plot illustrating the trend of the characteristic curves between $\nabla P_{A}=6.66 \times 10^{7} \mathrm{~Pa} / \mathrm{m}$ and $\nabla P_{B}=6.66 \times 10^{8} \mathrm{~Pa} \mathrm{~m}^{-1}$, showing that $Q(\nabla P)$ actually scales as $\nabla P^{\frac{1}{n}}$ for all the three bundles of capillaries. The slopes of the obtained straight lines are, respectively, 1.677 (equilateral triangular cross-sections), 1.678 (square crosssections) and 1.678 (circular cross-sections) and are very close to the expected value $\frac{1}{n}=\frac{1}{0.6} \sim 1.667$.

Finally, when dealing with the measurements obtained during a real YSM experiment on an unknown porous sample, the bundle of capillaries model used for the inversion procedure is expected to impact the obtained PSD, as mentioned above. To illustrate this effect, the numerically generated $Q(\nabla P)$ curve corresponding to the bundle of circular capillaries (red curve in Fig. 4) is considered. Those data are processed using the inversion method presented by Rodríguez de Castro et al. (2016, 2018), successively with a bundle composed of capillaries with circular, square and triangular cross-sections. The key stages of PSD calculation algorithm used in YSM are summarized in the following lines. During a YSM test, a Herschel-Bulkley fluid is injected through the investigated porous medium at $N+1$ different flow rates $Q_{j}(j=1 \ldots N+1)$, and the steady-state pressure gradient $\nabla P_{j}$ corresponding to each value of $Q_{j}$ is measured. Assuming a bundle of capillaries model with horizontal cylindrical pores, the pore-size class representing the radius of the newly incorporated pores contributing to the total flow rate $Q_{j}$ under a given pressure gradient $\nabla P_{i}$ is defined as:

$$
r_{i}=\alpha \frac{2 \tau_{0}}{\nabla P_{i}} \quad(i=1 \ldots N)
$$

The value of $\alpha$ in the preceding equation must be strictly greater than unity, because the contribution of the pores with $r=\frac{2 \tau_{0}}{\nabla P_{i}}(\alpha=1)$ is not noticeable at $\nabla \mathrm{P}_{\mathrm{i}}$. Rodríguez de Castro et al. $(2014,2016,2018)$ described the procedure used to determine the value of $\alpha$. In the YSM inversion method, the number of pores $n_{i}$ belonging to each pore radius class $r_{i}$ is calculated as being the maximum positive value respecting all the following conditions:

$$
Q_{s} \geq \sum_{g=1}^{i} n_{g} q\left(\nabla P_{s}, r_{g}\right) \quad \text { for } \quad s \geq i+1
$$

In the preceding equation, $q\left(\nabla P_{s}, r_{g}\right)$ is the volumetric flow rate of a Herschel-Bulkley fluid through a cylindrical capillary of radius $r_{g}$ under a pressure gradient $\nabla P_{s}$ (expression given by Skelland 1967 and Chhabra and Richardson 2008). The determination of $n_{i}$ starts 


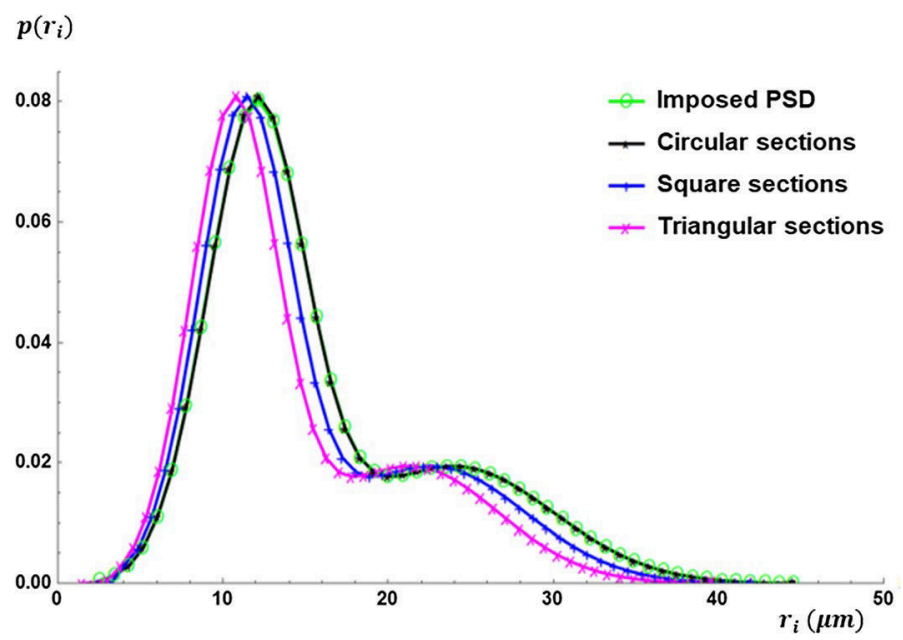

Fig. 5 Comparison of the PSD obtained by performing the YSM inversion based on bundles with capillaries of circular, square and triangular cross-sections

from $n_{1}$ (the number of largest pores) and then continues with $n_{2}, n_{3}$, etc., until the number of smallest pores $n_{N}$ is calculated.

It is important to keep in mind that for both square and triangular cross-sections, the inversion process is only made possible by using the empirical Eq. (13) proposed in the current work for capillaries of non-circular section. The obtained PSDs are presented in Fig. 5. These PSDs correspond to the probability of each representative pore class in terms of its frequency, which is defined by $p\left(r_{i}\right)=n_{i} / \sum_{j=1}^{N} n_{j}$, where $N$ is the total number of representative pore classes.

As can be observed, for all cross-sectional shapes, the global bimodal shape of the initial PSD is entirely reconstructed by the inversion procedure. Naturally, the inversion with the bundle of capillaries having a circular cross-section yields the initially imposed PSD and the model chosen to perform the inversion is seen to have a non-negligible impact on the obtained PSD. Indeed, it is observed that by using the modeled bundles of capillaries with square and triangular cross-sections, the PSD obtained with YSM presents pore sizes slightly smaller than the ones obtained by using the classical bundle of capillaries with circular cross-sections. This may be related to the fact that the flow rate increases when going from the circular to square and finally to triangular cross-section for any given pressure gradient when considering capillaries with the same inscribed circle (Fig. 4), as mentioned above. Moreover, since the contribution of each representative pore class to flow rate is taken as $n_{i} \cdot q\left(\nabla P_{i}, r_{i}\right)$ in the YSM inversion method, smaller values of $n_{i}$ are obtained for triangular and square cross-sections as compared to the traditional circular cross-section.

A criterion should be adopted for selecting the suitable pore shape to be used in the YSM inversion algorithm, based on the shape factor of the pores present in the analyzed porous sample. The shape factor of a single pore is defined as the ratio of cross-sectional area to the perimeter raised to the power of two (Øren and BakkE, 2002; López et al. 2003) and its value is $\frac{1}{4 \pi}$ for circular pores, $\frac{1}{16}$ for square pores and is comprised between 0 and $\frac{\sqrt{3}}{36}$ for triangular pores. The knowledge of the general form of the pore cross-section for a class of porous media can be a guide for the inversion process used in YSM. However, it should be noted that the distribution of shape factors in a porous sample is unknown unless some 
pore-network modeling is performed based on a digital image of the investigated material. In this regard, López et al. (2003) and López (2004) extracted the pore-network models of a Berea sandstone, a heterogeneous sandstone and a sand pack, showing that most pores were triangular. Therefore, on the basis of these results, triangular shape cross-sections can be assumed when applying YSM to these types of porous media.

\section{Conclusions}

In the present work, empirical flow rate/pressure gradient relationships for the flow of yield stress fluids through rectilinear capillaries with an equilateral triangle or a square as the cross-sectional shape have been proposed. The development is based on the idea that the flow rate of Herschel-Bulkley fluids flowing through these types of rectilinear capillaries under an imposed pressure gradient can be written as the product of two independent terms. The first one is the shape coefficient, based on the rigorous solution of the flow of Newtonian fluids through rectilinear capillaries. The second term, which contains the flow features, is obtained by including a critical Bingham number in the usual analytical flow rate/pressure gradient relationships of yield stress fluids through cylindrical capillaries. Such Bingham number accounts for the characteristic threshold length $l^{*}$ marking the onset of the flow. The current results also apply for the particular case of a Bingham fluid $(n=1)$.

The reliability of the proposed formulas has been assessed by comparison with simulation data coming from the literature (Malvault et al. 2017), as well as with the current numerical simulations performed in the three geometries within a wide range of flow rates. However, it must be noted that Reynolds numbers must be kept low enough to satisfy creeping flow conditions, which is the main assumption for this study. This assumption is realistic in the case of typical yield stress fluids such as polymer solutions or emulsions, which exhibit high values of shear viscosity leading to low values of Reynolds numbers in common applications.

Moreover, the interest of using these empirical flow rate/pressure gradient relationships in the context of yield stress fluid porosimetry (YSM) has been evinced. In this regard, the YSM inversion (Rodríguez de Castro 2014; Rodríguez de Castro et al. 2014) of a set of $Q(\nabla P)$ data generated with a bundle of cylindrical capillaries following a bimodal PSD has been shown to be highly accurate when the circular cross-sections expression is used. Furthermore, the obtained PSD when assuming either square or triangular cross-sectional shapes in the inversion algorithm have proved to be consistent.

A criterion for the choice of the cross-sectional shape when performing the YSM inversion still needs to be established. Indeed, studies in which porous samples are characterized by imaging techniques often reveal pores shapes with low circularity values (Gundogar et al. 2016; Li et al. 2018; Lai et al. 2018). In those studies, real pores are commonly assimilated to rectilinear capillaries with circular, square, triangular (and even rectangular) cross-sections. Therefore, additional data coming from microscopic images of thin sections or from previous knowledge of the shape of the solid grains (especially for unconsolidated porous media) can be used, when available, to guide the choice of the cross-section in YSM inversion. However, it can be anticipated from the present results that considering bundles of capillaries with square and equilateral triangle cross-section shapes will change the manner in which YSM experimental data are processed, affecting the obtained poresize distributions. 
Taking into account the non-circularity of the capillaries cross-sectional shape in the procedure of YSM inversion for the processing of experimental data is a first perspective of this work. As another perspective, the representativeness of the model underlying the inversion may be further improved by incorporating the converging-diverging character of pores. In this respect, Malvault et al. (2017) showed that the abrupt change of the crosssection size has a noticeable impact on the simulated flow rate at a given pressure gradient for Bingham fluids flow, independently of the shape of the cross-section. This impact is a consequence of changes in both the location and the shape of the yield surface (Burgos and Alexandrou 1999; Jay et al. 2002; Alexandrou et al. 2001). Nevertheless, the derivation of relevant approximations of flow rate/pressure gradient relationships in such geometries that are readily usable in YSM still remains an open research topic. Finally, the results of this work may also contribute to the study of the flow of yield stress fluids in pore-network models.

Authors' contributions Data collection, visualization and original draft preparation were performed by Terence Emery Mackaya. Azita Ahmadi-Sénichault, Abdelaziz Omari and Antonio Rodríguez de Castro contributed to the writing, reviewing and editing. All authors contributed to the conceptualization, methodology, investigation and analysis of the results.

Funding Not applicable.

Availability of data and Material The data and the details on the used material are available upon request.

\section{Compliance with Ethical Standards}

Conflict of interest The authors declare that they have no conflict of interest.

Code Availability Not applicable.

\section{References}

Alexandrou, A.N., McGilvreay, T.M., Burgos, G.: Steady Herschel-Bulkley fluid flow in threedimensional expansions. J. Nonnewton. Fluid Mech. 100(1), 77-96 (2001)

Ambari, A., Benhamou, M., Roux, S., Guyon, E.: Distribution des tailles des pores d'un milieu poreux déterminée par l'écoulement d’un fluide à seuil. C.R. Acad. Sci. Paris 311(2), 1291-1295 (1990)

Burgos, G.R., Alexandrou, A.N.: Flow development of Herschel-Bulkley fluids in a sudden threedimensional square expansion. J. Rheol. 43(3), 485-498 (1999)

Burlion, N., Bernard, D., Chen, D.: X-ray microtomography, application to microstructure analysis of a cementitious material during leaching process. Cem. Concr. Res. 36, 346-357 (2006)

Chhabra, R.P., Richardson, J.F.: Non-Newtonian Flow and Applied Rheology: Engineering Applications. Butterworth-Heinemann, Amsterdam (2008)

Damianou, Y., Georgiou, G.C.: Viscoplastic poiseuille flow in a rectangular duct with wall slip. J. Nonnewton. Fluid Mech. 214, 88-105 (2014)

Damianou, Y., Philippou, M., Kaoullas, G., Georgiou, G.C.: Cessation of viscoplastic poiseuille flow with wall slip. J. Nonnewton. Fluid Mech. 203, 24-37 (2014)

Damianou, Y., Kaoullas, G., Georgiou, G.C.: Cessation of viscoplastic poiseuille flow in a square duct with wall slip. J. Nonnewton. Fluid Mech. 233, 13-26 (2016)

Rodríguez de Castro, A.: Expériences d'écoulement de fluides à seuil en milieu poreux comme nouvelle méthode de porosimétrie. Ph.D. thesis, Ecole nationale supérieure d'arts et métiers-ENSAM, France (2014)

Rodríguez de Castro, A., Omari, A., Ahmadi-Sénichault, A., Bruneau, D.: Toward a new method of porosimetry: principles and experiments. Transp. Porous Media 101(3), 349-364 (2014) 
Rodríguez de Castro, A., Ahmadi-Sénichault, A., Omari, A., Savin, S., Madariaga, L.-F.: Characterizing porous media with the yield stress fluids porosimetry method. Transp. Porous Media 114(1), 213-233 (2016)

Rodríguez de Castro, A., Ahmadi-Sénichault, A., Omari, A.: Using xanthan gum solutions to characterize porous media with the yield stress fluid porosimetry method: robustness of the method and effects of polymer concentration. Transp. Porous Media 122(2), 357-374 (2018)

Gundogar, A., Ross, C., Akin, S., Kovscek, A.: Multiscale pore structure characterization of middle east carbonates. J. Petrol. Sci. Eng. 146, 570-583 (2016)

Herschel, W.H., Bulkley, R.: Konsistenzmessungen von gummi-benzollösungen. Colloid Polym. Sci. 39(4), 291-300 (1926)

Houston, A.N., Otten, W., Falconer, R., Monga, O., Baveye, P.C., Hapca, S.M.: Quantification of the pore size distribution of soils: assessment of existing software using tomographic and synthetic 3D images. Geoderma 299, 73-82 (2017)

Huilgol, R.R.: A systematic procedure to determine the minimum pressure gradient required for the flow of viscoplastic fluids in pipes of symmetric cross-section. J. Nonnewton. Fluid Mech. 136(2-3), 140-146 (2006)

Jay, P., Magnin, A., Piau, J.M.: Numerical simulation of viscoplastic fluid flows through an axisymmetric contraction. J. Fluids Eng. 124(3), 700-705 (2002)

Kefayati, G., Huilgol, R.: Lattice boltzmann method for the simulation of the steady flow of a bingham fluid in a pipe of square cross-section. Eur. J. Mech.-B/Fluids 65, 412-422 (2017)

Lai, J., Wang, G., Wang, S., Cao, J., Li, M., Pang, X., Zhou, Z., Fan, X., Dai, Q., Yang, L., et al.: Review of diagenetic facies in tight sandstones: diagenesis, diagenetic minerals, and prediction via well logs. Earth-Sci. Rev. 185, 234-258 (2018)

Li, Z., Wu, S., Xia, D., He, S., Zhang, X.: An investigation into pore structure and petrophysical property in tight sandstones: a case of the Yanchang formation in the Southern Ordos Basin, China. Mar. Pet. Geol. 97, 390-406 (2018)

López, X.: Pore-scale modelling of non-newtonian flow. Ph.D. thesis. Imperial College London (2004)

López, X., Valvatne, P.H., Blunt, M.J.: Predictive network modeling of single-phase non-Newtonian flow in porous media. J. Colloid Interface Sci. 264(1), 256-265 (2003)

Malvault, G.: Détermination expérimentale de la distribution de taille de pores d'un milieu poreux par l'injection d'un fluide à seuil ou par analysefréquentielle, $\mathrm{PhD}$ thesis, Arts et Métiers ParisTech (2013).

Malvault, G., Ahmadi, A., Omari, A.: Numerical simulation of yield stress fluid flow in capillary bundles: influence of the form and the axial variation in the cross section. Transp. Porous Media 120(2), 255270 (2017)

Mosolov, P., Miasnikov, V.: Variational methods in the theory of the fluidity of a viscous-plastic medium. J. Appl. Math. Mech. 29(3), 545-577 (1965)

Mosolov, P., Miasnikov, V.: On stagnant flow regions of a viscous-plastic medium in pipes. J. Appl. Math. Mech. 30(4), 841-854 (1966)

Mosolov, P., Miasnikov, V.: On qualitative singularities of the flow of a viscoplastic medium in pipes: PMM vol. 31, no. 3, 1967, pp. 581-585. J. Appl. Math. Mech. 31(3), 609-613 (1967)

Øren, P.-E., Bakke, S.: Process based reconstruction of sandstones and prediction of transport properties. Transp. Porous Media 46, 311-343 (2002)

Panaseti, P., Georgiou, G.C.: Viscoplastic flow development in a channel with slip along one wall. J. Nonnewton. Fluid Mech. 248, 8-22 (2017)

Papanastasiou, T.C.: Flows of materials with yield. J. Rheol. 31(5), 385-404 (1987)

Patzek, T., Silin, D.: Shape factor and hydraulic conductance in noncircular capillaries: I. One-phase creeping flow. J. Colloid Interface Sci. 236(2), 295-304 (2001)

Peng, S., Zhang, T., Loucks, G.L., Shultz, J.: Application of mercury injection capillary pressure to mudrocks: conformance and compression corrections. Mar. Pet. Geol. 88, 30-40 (2017)

Roquet, N., Saramito, P.: An adaptive finite element method for viscoplastic flows in a square pipe with stick-slip at the wall. J. Nonnewton. Fluid Mech. 155(3), 101-115 (2008)

Saramito, P., Roquet, N.: An adaptive finite element method for viscoplastic fluid flows in pipes. Comput. Methods Appl. Mech. Eng. 190(40-41), 5391-5412 (2001)

Skelland, A.H.P.: Non-Newtonian Flow and Heat Transfer. Wiley, New York (1967)

Wildenschild, D., Sheppard, A.P.: X-ray imaging and analysis techniques for quantifying pore-scale structure and processes in subsurface porous medium systems. Adv. Water Resour. 51, 217-246 (2013)

Zhu, H., Kim, Y., De Kee, D.: Non-Newtonian fluids with a yield stress. J. Nonnewton. Fluid Mech. 129(3), 177-181 (2005) 